\title{
Kikuchi-Fujimoto Disease Associated to Connectivitis -About Two Cases-
}

\section{Nesrine Regaïeg ${ }^{1}$, Mayeda Ben Hamad ${ }^{1}$, Nesrine Belgacem ${ }^{1}$, Hassen Baïli ${ }^{1}$, Najla Lassoued ${ }^{1}$ Salem Bouomrani* ${ }^{1 *}$}

${ }^{1}$ Department of Internal medicine, Military Hospital of Gabes, Gabes 6000, Tunisia. salembouomrani@yahoo.fr

*Corresponding Author: Pr Salem Bouomrani, Department of Internal medicine, Military Hospital of Gabes, Gabes 6000. Tunisia.

\section{Abstract}

Introduction: Kikuchi-Fujimoto's disease (KFD) or histiocytic necrotizing lymphadenitis is a rare clinico-pathological entity of unknown etiology that primarily affects young Asian women. The association of this disease with dys-immune systemic disease is rare. We report two cases.

Observation 1: 28 year old woman, having systemic lupus erythematosus (SLE) with cutaneous, hematologic, and renal complication, who developed six years after cervical and axillary febrile lymphadenopathy. A lymph node biopsy showed histological and Immunohistochemical aspects suggesting KFD.

Observation 2: 30 year old woman, without medical history was hospitalized for febrile superficial poly -lymphadenopathy. Etiological investigation was negative. Lymph node biopsy confirmed the diagnosis of KFD. Two years later primary Sjogren's syndrome was diagnosed in this patient.

Favorable evolution was noted in both cases after 1-2 months.

Conclusion: KFD is a very rare histological diagnosis. Underlying autoimmune conditions are rarely reported; particularly the association with primary Sjogren's syndrome is exceptional. These associations represent a real diagnostic challenge and they deserve to be known by physicians.

Keywords: Kikuchi-Fujimoto's disease; histiocytic necrotizing lymphadenitis; systemic lupus erythematosus ; primary Gougerot-Sjögren syndrome.

\section{INTRODUCTION}

Kikuchi-Fujimoto syndrome (KFD) is a rare anatomo -clinical entity with an imprecise pathogenesis and recent individualization since its first description dates back to 1972 in Japan, where it was reported simultaneously by the two authors Kikuchi M. [1] ] and Fujimoto Y. [2]. His diagnosis remains histological characterized by histiomonocytic hyperplasia with ganglionic necrosis thus defining the necrotizing histiocytic lymphadenitis (NHS); scientific name of this syndrome [3].

It affects young people under the age of thirty, most of them female and mainly of Asian origin [3]. It remains exceptional among Caucasians.

The association of KFD with other systemic dysimmunitary diseases is rare and poses diagnostic problems that sometimes represent a real challenge for the clinician [3-6]. This association suggests similar etiopathogenic mechanisms including a common autoimmune signature [7].

We report two particular cases of this syndrome associated, for the first time, with systemic lupus erythematosus (SLE) and primary Sjögren's syndrome (PSS) for the second; Association exceptionally reported previously.

\section{Case Report 1}

28-year-old woman, followed since age 22 years for SLE meeting the ACR criteria, with cutaneous involvement: photosensitivity, erythema in vespertilio andoropharyngealulceration, hematologic:haemolytic anemia at 9g / l with a positive direct Coombs test, leucopenia at 3900 / mm and lymphopenia at 1200 / 
Kikuchi-Fujimoto Disease Associated to Connectivitis -About Two Cases-

$\mathrm{mm}$, and renal: microscopic hematuria, proteinuria at $2.5 \mathrm{~g} / 24 \mathrm{~h}$ and renal biopsy lupus nephropathy stage III: segmental glomerulonephritis and focal.

She was treated with hydroxychloroquine and prednisone at full dose $(1 \mathrm{mg} / \mathrm{kg} / \mathrm{d})$ for six weeks followed by a gradual decrease to a dose of $10 \mathrm{mg}$ / d with a favorable course. She was admitted six years after the diagnosis of SLE for fever, asthenia and inflammatory arthralgia of recent evolution. The physicalexaminationfoundmultiplelymphadenopathy, not very sensitive, mobile and bilateral cervical and axillary seat.

The infectious balance, in particular tuberculosis, was negative.

Lymph node biopsy showed follicular hyperplasia, histiocytic and monocytic infiltrate with ganglionic necrosis and positive immuno-labeling by anti-CD68 compatible with SKF diagnosis. This syndrome was associated with an outbreak of lupus disease (disease activity index «SLADAI» at 15). The evolution under systemic corticosteroid therapy at a dose of $0.5 \mathrm{mg} / \mathrm{kg}$ / day for one month followed by a progressive decrease was favorable with disappearance of functional signs in the first week and lymphadenopathy after one month.

\section{Case Report 2}

A30-year-old woman with no pathological history who had been hospitalized for poly febrile adenopathy for 15 days. The examination was normal, except for the presence of multiple cervical adenopathies posterior, axillary and inguinal mobile, sensitive and without fistulization.

The etiologic assessment was negative, eliminating particularly active tuberculosis, solid neoplasia and underlying malignant hematological malignancy. This assessment included a blood count, biological markers of inflammation, electrophoresis of serum proteins, liver enzymes, a calcium phosphate balance with angiotensin-converting enzyme assay, tumor markers, the search for antibodies ant nuclear, tuberculin intradermal reaction, sputum BK and gastric tubing, chest x-ray, abdominal ultrasound, thoracoabdominopelvic computed tomography, blood smear, blood cultures and serodiagnosis of mononucleosis Infectious Disease, Toxoplasmosis and Human Immunodeficiency Viruses 1 and 2.

Lymph node biopsy showed histological signs of necrotizing histiomonocytic lymphadenitis and positive labeling by anti-CD68 compatible with SKF diagnosis. No malignant cells or lymphocytic atypia were noted. The evolution was spontaneously favorable with apyrexia after one week and disappearance of adenopathies after two months.

Two years later, the diagnosis of primary Sjögren's syndrome was made in this patient with the following signs: xerostomia, xerophthalmia, pathological ocular tests, stage III chronic lymphocytic sialadenitis in the biopsy of accessory salivary glands, positive antinuclear, anti-SSA, and anti-SSB antibodies.

\section{Discussion}

Considered to be the clinical expression of an inadequate immune response resulting from endogenous hyperstimulation limited to lymphoid tissue $[3,8]$, SKF presents clinically as a polyadenopathy febrile, most often cervical posterior [3]. Other ganglionic territories are rarely affected; especially the axillary and inguinal areas.

In this classic clinical picture, splenomegaly, hepatomegaly with disruption of biological liver tests, lymphopenia, arthralgia, rash and weight loss may be associated to varying degrees $[3,9]$. All these manifestations signify the systemic nature of this affection.

Usually the evolution is towards healing after one to three months, episodes of recurrence can exceptionally be at intervals varying to several years $[3,8,9]$.

The association of a SKF with systemic and / or organspecific dys-immune diseases is rarely reported: the large personal series of Sopeña B. et al., Of 20 patients with SKF over a period of twenty years (1990-2010), found an associated dys-immune disease in nine patients: SLE (4 cases), PSS (2 cases), primary antiphospholipid syndrome (1 case), lupus-like syndrome (1 case) and leukocytoclastic vasculitis (2 cases) [4]. Other rarer associations were reported as sporadic cases: myasthenia [7], autoimmune hepatitis [5], juvenile chronic arthritis [10], adult Still's disease [11], inflammatory myopathies [12] and haemophagocytic syndrome [13].

KFD may be the first manifestation of underlying systemic disease [14], and the existence during this syndrome of cytopenias as well as the female sex of the patients, and the painful nature of lymphadenopathy are significantly predictive of such coexistence [4].

On the other hand, the presence of an underlying dysimmune disease is a predictor of recurrence for SKF, even at intervals of several years [15]. 
Kikuchi-Fujimoto Disease Associated to Connectivitis -About Two Cases-

These associations emphasize the SKF's dys-immune character and suggest for several authors an autoimmune hypothesis at the origin of this syndrome [7].

Although the coexistence of KFD and lupus disease is still described as rare [3,5], SLE is the most common dys-immune disease associated with this syndrome; in fact, the large meta-analysis of the world literature made by Kucukardali Y. et al., in 2007 and including the 244 cases of KFD published until that date, found an association with SLE in 32 patients, or 13.11\% [6 ].

KFD-SLE was reported mainly for the systemic form of lupus $[8,16]$ and more rarely for chronic discoid lupus [8].

This association poses diagnostic and therapeutic problems; in fact, a poly febrile adenopathy in a lupus patient on corticosteroid or immunosuppressive treatment must first evoke tuberculosis, particularly in countries like ours, where this infection is still endemic [16]. This is important to know especially as the association of a SKF with a progressive pulmonary tuberculosis can be seen! [17].

On the other hand, lupus lymphadenitis can make a differential diagnosis with a SKF associated with lupus because it can also contain zones of necrosis [18]. Hence the interest of the histology, which is the only investigation allowing the diagnosis of certainty by showing the absence or the scarcity of the cytotoxic $\mathrm{T}$ lymphocytes usually abundant in the KFD and the immuno-marking of antibody CD68 which is very specific to KFD [3].

Finally it should be noted that an isolated SKF can give a malar eruption simulating that of SLE [19] which represents a real diagnostic challenge for clinicians.

For the PSS, association with a KFD is more exceptional: until today only five observations have been found [4, $15,20-22]$. Our observation is to our knowledge the sixth reporting this association.

The main differential diagnosis during this association, and particularly in the diffuse forms of KFD arises with lympho-proliferative syndromes (mainly malignant lymphomas) and with ganglionic tuberculosis. These two differential diagnoses are important to eliminate because:

- The lymphomatous transformation remains the main complication to be feared during a primary SGS and moreover the association of a KFD to diffuse large cell lymphoma was reported [23].
- The differential diagnosis of KFD with ganglionic tuberculosis also represents a real dilemma [24], especially since necrotizing histiocytic lymphadenitis (KFD) may precede active visceral tuberculosis (especially pleural) as illustrated by the observation of Kwon HH et al. [25].

Careful histological study and immunoblotting are of interest in these situations.

\section{ConCLUSION}

As rare as they are, these associations are known. This knowledge will in many cases avoid several unnecessary investigations, sometimes very laborious and expensive, to eliminate, in front of an apparently isolated SKF, an infectious cause or malignant lymphoma.

\section{Abbreviations}

SLEDAI: Systemic Lupus Erythematosus Disease Activity Index (indice d'activité de la maladie lupique)

CD68: Cluster of Differentiation 68 (Membrane glycoprotein specific for monocytes / macrophages)

\section{REFERENCES}

[1] Kikuchi M. Lymphadenitis showing focal reticulum cell hyperplasia with nuclear debris and phagocytes: a clinicopathological study. Acta Hematol Jpn. Nippon Ketsueki Gakkai Zasshi. 1972; 35: 379-80.

[2] Fujimoto Y, Kjima Y, Yamaguchi K. Cervical subacute necrotizing lymphadenitis: a new clinicopathologic entity. Naika. 1972; 20: 920-7.

[3] Ruaro B, Sulli A, Alessandri E, FraternaliOrcioni G, Cutolo M. Kikuchi-Fujimoto's disease associated with systemic lupus erythematous: difficult case report and literature review. Lupus. 2014;23(9):939-944.

[4] Sopeña B, Rivera A, Vázquez-Triñanes C, Fluiters E, González-Carreró J, del Pozo M et al. Autoimmune manifestations of Kikuchi disease. Semin Arthritis Rheum. 2012;41(6):900-6.

[5] Shusang V, Marelli L, Beynon H, Davies N, Patch D, Dhillon AP et al. Autoimmune hepatitis associated with Kikuchi-Fujimoto's disease. Eur J Gastroenterol Hepatol. 2008;20(1):79-82.

[6] Kucukardali Y, Solmazgul E, Kunter E, Oncul O, Yildirim S, Kaplan M. Kikuchi-Fujimoto Disease: analysis of 244 cases. Clin Rheumatol. 2007;26(1):50. 
Kikuchi-Fujimoto Disease Associated to Connectivitis -About Two Cases-

[7] Onasanya O, Nochlin D, Casas V, Peddareddygari LR, Grewal RP. Kikuchi-fujimoto disease associated with myasthenia gravis: a case report. Case Rep Med. 2010;2010. pii: 903252. doi: 10.1155/2010/903252. [Epub 2010 Aug 11].

[8] Lecoues S, Michel M, Zarrouk Y, Ganulard P, Schaeffer A, Godeau B. Recurrent Kikuchi's disease in patient with discoid lupus: Rev Med Interne. 2003; 24(9):613-6.

[9] Dokic M, Begovic V, Bojic I, Tasic O, Stamatovic D. Kikuchi-Fujimoto disease: Vojnosanit Pregl. 2003; 60(5):625-30.

[10] Ramanan AV, Wynn RF, Kelsey A, Baildam EM. Systemic juvenile idiopathic arthritis, Kikuchi's disease and haemophagocytic lymphohistiocytosis-is there a link? Case report and litterature review: Rheumatology (oxford). 2003; 42(4):596-8.

[11] Cousin F, Grezard P, Roth B, Gregoire-Barled M, Perrot H. Kikuchi disease associated with Still disease: Int J Dermatol. 1999; 38(6): 464-7.

[12] Wilkinson CE, Nichol F. Kikuchi-Fujimoto disease associated with polymyositis: Rheumatology (oxford). 2000; 39(11):1302-4.

[13] Kim YM, Lee YJ, Nam SO, Park SE, Kim JY, Lee EY. Hemophagocytic syndrome associated with Kikuci's disease: J Korean Med Sci. 2003;18(4):592-4.

[14] Lu S, Zhang J, Zhou W, Wang XM. Histiocytic necrotic lymphadenitis as an initial presentation in two children with Sjogren's syndrome and/or systemic lupus erthematous. Zhongguo Dang Dai Er Ke Za Zhi. 2010;12(4):311-2.

[15] Bogusz AM, Bhargava P. Recurrent histiocytic necrotizing lymphadenitis with a long latency in a patient with autoimmunity: a case report and review of literature. Int J Surg Pathol. 2013;21(3):287-96.
[16] Adhikari RC, Sayami G, Lee MC, Basnet RB, Shresta PK, Shrestha HG. Kikuci-Fujimoto disease in Nepal: a study of 6 cases: Arch pathol lab Med. 2003; 127(10):1345-8.

[17] Velasco Malagam MJ, Zambrana Garcia JL, Adarraga Cansino MD, Rosa Jimenez F. Disease of Kikuchi-Fujimoto and pulmonary tuberculosis, an infrequent association: Rev Clin Esp. 2003; 203(10):508-9.

[18] Hu S, Kuo T, Hong HS. Lupus lymphadenitis simulating Kikuchi's lymphadenitis in patients with systemic lupus erythematosus: a clinicopathological analysis of six case and review of the litterature: Pathol Int. 2003; 53(4):221-6.

[19] Okuzawa C, Kuroiwa T, Kaneko Y, Veki k, Tsukada Y, Nojima Y. Kikuchi disease accompanied lupus-like butterfly rash: J Rheumatol. 2003; 30(4):857-9.

[20] Ogata S, Bando Y, Saito N, Katsuoka K, Ishii M. Kikuchi-Fujimoto disease developed into autoimmune disease: a report of two cases. Mod Rheumatol. 2010;20(3):301-5.

[21] Soy M, Peynirci H, Bilgi S, Adali MK, Güresci S. Kikuchi-Fujimoto disease coexisted with Sjogren's syndrome. Clin Rheumatol. 2007;26(4):607-8.

[22] Miyashita Y, Yamaguchi M, Fujimoto W.Painful indurated erythema suggestive of KikuchiFujimoto disease in a patient with primary Sjögren's syndrome. J Dermatol. 2003;30(8):608-11.

[23] Astudillo L. La maladie de Kikuchi-Fujimoto. Rev Med Interne. 2010;31(11):757-65.

[24] Pai VD, Jadhav RR. Kikuchi's Disease: A Diagnostic Dilemma. Indian J Surg. 2015;77(Suppl 1):164-5.

[25] Kwon HH, Kim SK. Histiocytic necrotizing lymphadenitis (Kikuchi-Fujimoto disease) preceding tuberculous pleurisy. Int J Rheum Dis. 2014;17(2):223-5.

Citation: Salem Bouomrani, et al. Kikuchi-Fujimoto Disease Associated to Connectivitis -About Two CasesOpen Access Journal of Internal Medicine. 2018; 1(1): 21-24.

Copyright: (c) 2018 Salem Bouomrani, et al. This is an open access article distributed under the Creative Commons Attribution License, which permits unrestricted use, distribution, and reproduction in any medium, provided the original work is properly cited. 